\title{
APPLICATION OF LOCATION INFORMATION BY STEREO CAMERA IMAGES TO PROJECT PROGRESS MONITORING
}

\author{
Soungho CHAE \\ Kajima Technical Research Institute \\ 2-19-1 Tobitakyu Chofu-shi, Tokyo, Japan \\ chae@kajima.com
}

\author{
Naruo KANO \\ Waseda University \\ 3-4-1Ookubo Shinjyukuku, Tokyo, Japan \\ kano@waseda.jp
}

\begin{abstract}
In this paper, the authors discussed the overview of a method for estimating the 3-D coordinated data for development of a project control system with stereo camera images. The information for control project with location information in the 3-dimensional space of time series from images is proposed. SFM (Structure From Motion) is used for collection of camera motion and 3-D object data. The image collection on actual construction site was performed and 3D structure from stereo camera images was evaluated. According to the result, the possibility of the monitoring and the factors necessary to development for practical were proposed.
\end{abstract}

\section{KEYWORDS}

Location Information, Stereo Camera, Image Processing, Project Control

\section{INTRODUCTION}

In order to control progress of a project, it is necessary to check the information of resources placement in construction site such as workers, parts, and heavy equipments. However, while measurement and record of the location of resources depend heavily on physical observation of supervisors, the information of resources placement is difficult to be reflected in timely control of progress. In many cases, the actual location of resources which administrators cannot check are assumed based on operating reports from sub-contractors. Site management in the construction project becomes more efficient by monitoring and evaluating the location of resources.

In recent years, many studies have indicated that the automatic data collection is efficiency in construction management with development of the technology, such as GPS, RFID, LADAR, and image processing [1]. The authors have suggested the method to measure the location of workers using RFID [2]. The location information is placed as a main data for improvement of construction management.

Image processing by cameras has possibility as an alternative means of person's physical observation in the location-aware technology. Camera is one of the sensors which can collect much information economically. In the construction site, the advantages of camera images as a source of actual information have been recognized [3] [4]. However, in many cases, it is used for the watch of scenes at remote locations. One of the reasons for dependence on visual appreciation is thought to be the technical problem for automatic recognition in all environments.

The objective of this research is application of location information to project management and verification of data collecting process with stereo camera images. A prototype with commercial software was used for experiment, and the image collection is undertaken in actual construction site. Then the 3-D coordinates was calculated from the images, and determined the factors necessary for location information in control project. 


\section{APPLICATION OF 3D LOCATION INFORMATION IN PROJECT CONTROL}

Installation progresses of construction are carried out on the site, and the each progress needs arrangement of resources such as workers, parts, and equipments according to actual condition for proper control of project. The workers are deployed in installation area to attach the stocked parts to the indicated place. The equipments are used in order to support handling, movement, remove, measurement, processing. Collecting of the time and position of resources in the level of task is required for the overall control of the project.

The real-time monitoring of location of each resource is useful to subjective judgment of experienced supervisors in process control. The estimation of condition of tasks and the formulation of problems using the location of resources are necessary. We can control the project progressively if we can collect how and where resources are performing. Also, it would be easier to make analysis for site management, such as safety, productivity and quality control.
Figure 1 shows control information on the tasks of each resource. In this paper, we defined the information as control information to control project. The control information is computed with location information in the 3-dimensional space of time series from images. For example, the installation interval from volume of parts, the moving distance of workers from 3-D coordinates, etc. are computed in time series.

A number of methods for collection of 3-D data of position in space have been developed. In this method, we examined the factors relating to the computation of control information and adopted stereo camera images. Although camera image processing has the technical problem for automatic recognition, it has the advantages that input data can be recognized visually and compiled heavily.

Many studies to reconstruct 3-D shape of the object from several images are contributed in the field of computer vision [5]. In this study, we used SFM (Structure From Motion) for collection of camera motion and 3-D object data. SFM estimates camera motions and reconstructs objects simultaneously based on epipolar geometry.

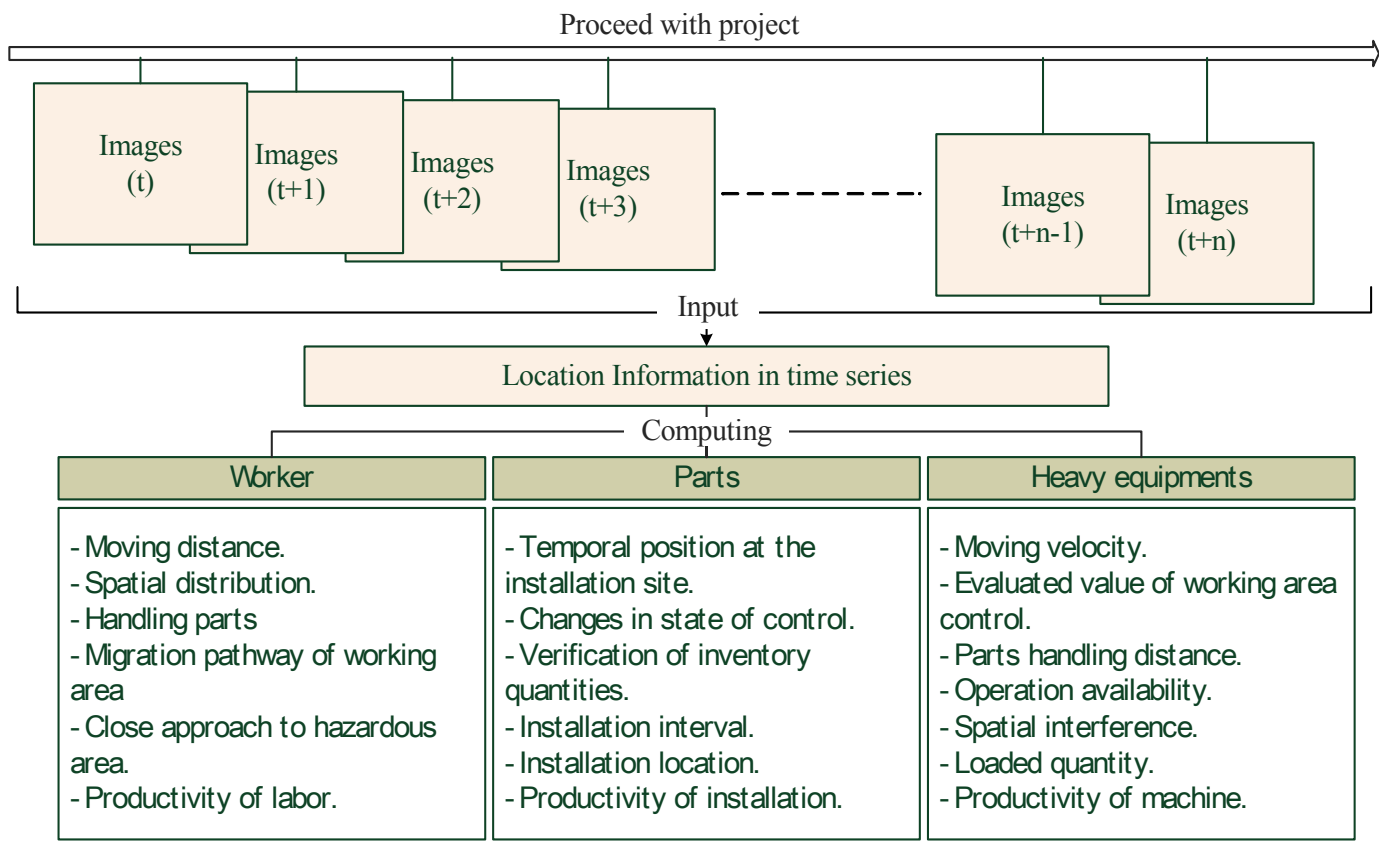

Figure 1 Computation of Control Information from Location Information 


\section{ACQUISITION OF 3D COORDINATE DATA WITH STEREO CAMERA IMAGES}

\subsection{Computing 3D Structure from Images}

In order to reconstruct objects from more than one image, it is necessary to perform the camera calibration which is estimation of camera motions. The camera calibration can be classified two methods according to using coordinated makers. When using coordinated makers, the high accuracy of calculation is obtained, but the effort for installing is required. The effort of calibrations increases in the construction site with much change of the camera position for avoiding obstacles.

In this study, we performed the camera calibration with corresponding image points without coordinated makers and computed the 3-D coordinated data of objects.

Fundamental matrix $\mathrm{F}$ can be computed from pixel coordinates of correspondence points in no calibrated camera images [6]. The 8-points algorithm is a method of computing the fundamental matrix F [7].

If $\mathrm{x}$ and $\mathrm{x}^{`}$ are corresponding image points, then the fundamental matrix $\mathrm{F}$ is unique $(3,3)$ rank 2 homogeneous matrix which satisfies Eq. (1). The fundamental matrix $\mathrm{F}$ is determined with more than 8 point correspondences (Figure2)

$$
x^{\prime T} F x=0
$$

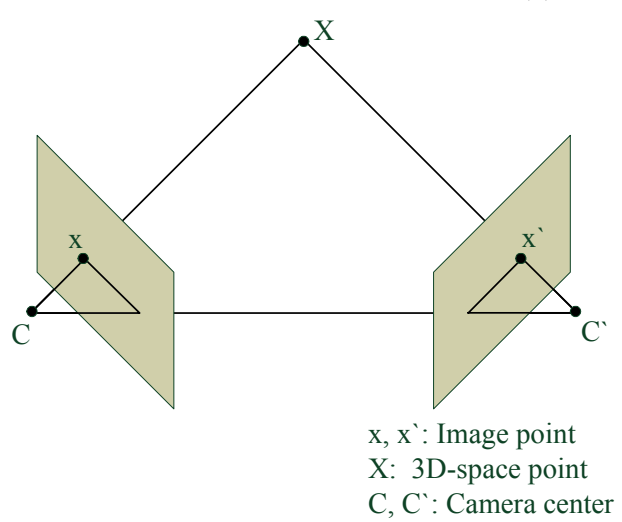

Figure 2 Point Correspondence Geometry

\subsection{Experimental result}

The authors performed the image collection on actual construction site in order to evaluate the 3D structure from stereo camera images. The images were obtained from installing underground structure and 3D coordinate data was computed with commercial software. The camera equipment used was HDV camcorder camera with digital video tape (Table1). We converted the digital video tape into AVI format suitable for computer image processing.

Table 1 Camera specifications

\begin{tabular}{c|c}
\hline Product nam e & Sony HVR-A 1J \\
\hline Num ber of pixels & $1440 * 1080$ \\
\hline A spect ratio $(\mathrm{W} \mathrm{H})$ & $16: 09$ \\
\hline Sensor type & $1 / 3$ inch CM OS \\
\hline
\end{tabular}

The two cameras were fixed on the tripods and installed on the roof of the neighbor building for film of the overall site. The installed height of camera is about 13 meters from first floor of construction site. Since the distance between two cameras influenced the calculation results, we set to about 10 meters as maximum allowable distance. The film was taken for one hour a day over five days. The posture and position of cameras was modified for everyday

Figure3 shows the results of camera calibration calculated with the corresponding image points of a day. We obtained 3D coordinate data of objects from calibrated camera images and computed the control information (Figure4)

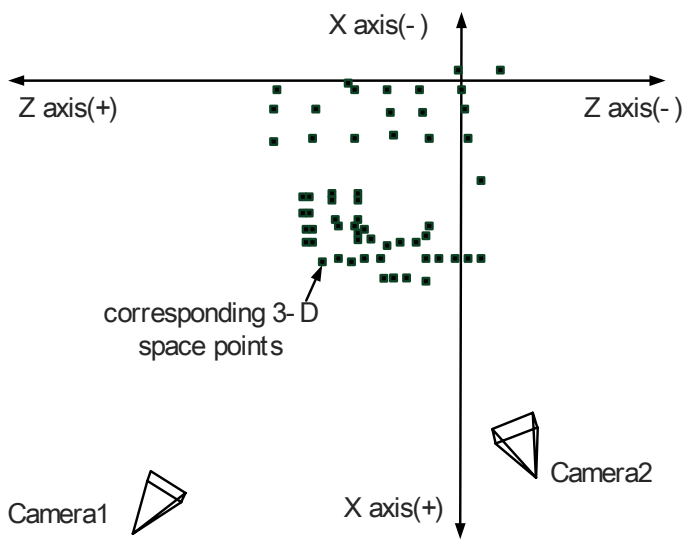

Figure 3 Results of camera calibration 


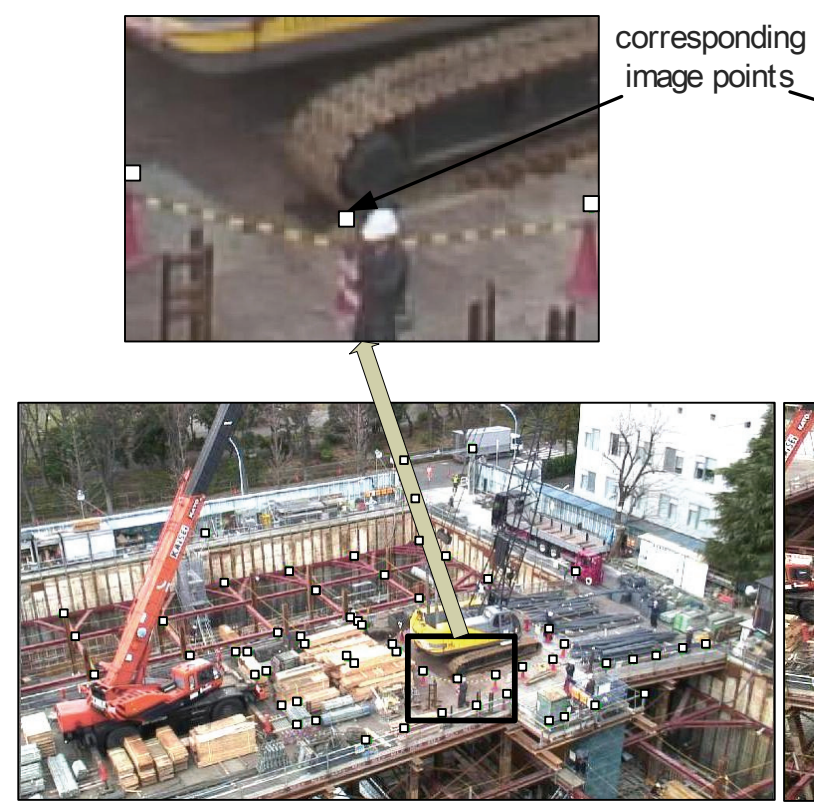

Camera1 image

Figure 4 Images of Each Camera and Corresponding Image Points

\section{CONCLUSION}

The authors examined the application of the 3-D coordinate data by stereo camera images to project progress control. The experiment was performed and camera calibration results for calculate 3-D structure was obtained

After the research, we found that increases in position error due to camera characteristic. Relative posture of cameras, image resolution, and optical distortion may result measurement noise.

In order to apply reconstruct objects technology by camera images into the project progress control in construction site, stabilization algorithm on automatic recognition of objects, development of optical noise reduction process, and improvement of hardware performance are necessary.

\section{ACKNOWLEDGMENT}

This work has been supported by the Grant-in-Aid for Scientific Research on Priority Areas (B) No.17360302 of Japan Society for the Promotion of Science (JSPS).

\section{REFERENCES}

[1] R.Navon, Research in automation measurement of project performance indicators, Automation in construction, 16(2007)176-188, 2007

[2] Soungho CHAE etc., An estimation method of working area with RFID technology, $5^{\text {th }}$ ICCPM $/ 2^{\text {nd }}$ ICCEM, 47(1-8), 2007

[3] J. Abeid etc., PHOTO-NET II : a computer based monitoring system applied to project management, Automation in construction, 12(2003)603-616, 2003

[4] Y. Wu and H. Kim, Digital imaging in assessment of construction project progress, 21th ISARC, 537-542, 2004

[5] R. Hartley and A. Zisserman, Multiple view geometry in computer vision $2^{\text {nd }}$ edition, Cambridge University press, 2003

[6] Q.T. Luong and O.D. Faugeras, The fundamental matrix: theory algorithms and stability analysis, Intl. Journal of Computer Vision, 17(1):43--76, 1996

[7] H.C.Longuet-higgins, A Computer algorithm for reconstructing a scene from two projections, nature, Vol.293, 133-135, 1981 